\title{
Alteraciones endometriales en el embarazo ectópico
}

\author{
Dr. Gerardo Chadid Samur \\ Dr. Juan Takano Moron
}

Hasta hace unos pocos años, se le concedía escaso valor al estudio del endometrio en el diagnóstico del embarazo ectópico ya que el criterio clásico de reacción decidual en ausencia de vellosidades coriales, como signo de probabilidad de embarazo ectópico, prese ta el inconveniente de su relativa baja incidencia y la dificultad b en conocida de diferenciarla de la reacción seudodecidual en endomctrios hipersecretores o el simple endometrio menstrual.

En el año de 1954 Arias Stella (1) del Perú describe alteraciones endometriales en abortos incomp?etos, mola, coriocarcinoma y un caso de embarazo ectópico, que según su propia expresión se caracterizan por "presencia de focos en la mucosa, constituidos por grunos glaudulares o glándulas aisladas, en los que se ven células de núcleo hipertrófico o gigante, hipercromáticos y de forma irregular".

"E1 citoplasma de estas células muestra por lo general inten a vacuolización secretoria y hay evidencia de simultánea actividad pro. liferativa, pues el epitelio se estratifica en varias capas, frecuentemente formando proyecciones intraluminarcs. No raramente se ven mitosis típicas o atípicas a este nivel. La pérdida de la polaridad es otro carácter distintivo de la lesión. El estroma que acompañ a estas glándulas atípicas puede o no mostrar reacción decidual. E? algunos casos se forman verdaderas áreas adenomatosas atípicas por la disposición apretada de las glándulas, en otros casos se pueden ver las glándulas normales, al lado de otras atípicas aisladas".

En el año 1957 aparecen los trabajos de Brux-Vaissade (2); P1 des-Wheeler (3) y Arias Stella-Gutiérrez (4) que describen estas 
mismas alteraciones en el endometrio de embarazos ectópicos. En 1958 Thorkild Frederiksen (5) describe como reacción Arias Stella las mismas alteraciones estudiando treinta y dos casos.

La incidencia de alteraciones dadas por estos autores, que comentaremos posteriormente, son inferiores a las obtenidas por nosotros debido posiblemente a diferencias en el material y métodos empleados, así como a la coordinación de los Departamentos de Obstetricia Ginecología y Patología de la Universidad del Valle.

Como habíamos mencionado, estas alteraciones han sido descritas en el aborto incompleto, la mola, el coriocarcinoma y el embarazo ectópico. Nosotros hemos tenido la oportunidad de estudiar el endometrio de cinco embarazos normales, en piezas quirúrgicas de histerectomías, en las que se desconocía la presencia de un embarazo y cuya indicación se produjo por verdaderos y falsos diagnósticos de leiomiomatosis. Todos los casos presentaban embarazos de 10 a 14 semanas y mostraron las mismas alteraciones descritas anteriormente.

Consideramos que el hallazgo de estas alteraciones, en ausencia de vellosidades coriales, es un auxiliar más en el difícil diagnóstico de embarazo ectópico, de gran valor cuando se ha descartado el embarazo intrauterino e inestimable cuando en una metrorragia de oscura etiología nos indica la existencia de tejido corial intra o extrau terino.

Creemos también que sus limitaciones deben ser bien conocidas, ya que es una alteración focal, que en nuestro material es muy frecuente pero que depende posiblemente de la vitalidad del tejido corial como parece demostrarlo el estudio de 3 casos de embarazos ectópicos con vellosidades hialinizadas en los que no se encontraron estas alteraciones.

\section{MATERIAL Y METODOS}

El material corresponde a 25 casos de embarazo ectópico tubario comprobados por estudio Anatomo-Patológico. En 22 casos se obtuvo endometrio por legrado practicados entre 25 y 0 dias antes de la intervención quirrúrgica. Los tres restantes se estudiaron en material de histerectomía con salpingo ooforectomía bilateral, en los casos que se encontraron ectópicos como hallazgo anatomopatológico.

Para el estudio de trompas y endometrio se utilizó inclusión en parafina y coloración con hematoxilina-eosina. Las preparaciones fueron estudiadas por ambos autores separadamente, obteniendo resultados muy similares.

Todos los casos correspondian a ectópicos tubáricos. 


\section{CRITERIOS DE CLASIFICACION}

Para resumir las alteraciones descritas las hemos agrupado en alteraciones morfológicas y alteraciones estructurales.

\section{ALTERACIONES MORFOLOGICA}

El criterio de clasificación morfológica ha sido formado por las siguientes alteraciones:

a) El aumento del tamaño de algunos núcleos del epitelio glandular, que contrastan con los vecinos; de formas variadas y que $\mathrm{p}$ esentan mitosis más o menos numerosas.

b) La pérdida de la polaridad celular.

c) El aumento del tamaño de los núcleos del epitelio glandu'ar en comparación con las glándulas vecinas.

d) La vacuolización del citoplasma de estas células.

\section{ALTERACIONES ESTRUCTURALES}

E1 criterio de clasificación estructural ha sido formado por las siguientes alteraciones:

a) Glándulas que muestran prolongaciones (espinas conju-tivas) hacia la luz glandular y que le dan un aspecto de sirrra de carpintero.

b) Marcada proliferación glandular que en algunos sitios le dan un aspecto adenomatoso por el escaso estroma.

c) Glándulas de luz amplia que no llegan a las formas quísticas.

d) La poliestratificación de los núcleos.

\section{RESULTADOS}

El cuadro siguiente muestra los resultados obtenidos con estos criterios. 


\section{Cuadro $\mathbf{N}^{\circ} 1$}

Morfología, Reacción Decidual y Alteraciones Estructurales de 25 Endometrios. Obtenidos 22 por Legrado y 3 por Histerectomía

Clínica de Maternidad de Cali y Hospital Departamental Universitario

\begin{tabular}{|c|c|c|c|c|c|}
\hline$\underset{\mathbf{N} ?}{\text { Caso }}$ & $\begin{array}{l}\text { Aspecto del } \\
\text { endometrio: }\end{array}$ & $\begin{array}{l}\text { Reacción } \\
\text { decidual }\end{array}$ & $\begin{array}{l}\text { Alteraciones } \\
\text { estructurales }\end{array}$ & $\begin{array}{l}\text { Alteraciones } \\
\text { morfológicas }\end{array}$ & $\begin{array}{c}\text { Trofob. en el } \\
\text { momento del } \\
\text { legrado }\end{array}$ \\
\hline 1 & Proliferativo & + & + & - & Activo \\
\hline 2 & Secretor & ++ & +++ & + & Activo \\
\hline 3 & Secretor & +++ & $+t+$ & ++ & Activo \\
\hline 4 & Proliferativo &.- & + & + & Activo \\
\hline 5 & Secretor & +++ & +++ & - & Activo \\
\hline 6 & Proliferativo & - & - & + & Poco activo \\
\hline 7 & Secretor & - & +++ & ++ & Activo \\
\hline 8 & Proliferativo & - & ++ & + & Activo \\
\hline 9 & Secretor & + & +++ & ++ & Activo \\
\hline 10 & Secretor & + & - & ++ & Activo \\
\hline 11. & Proliferativo & - & - & + & Activo \\
\hline 12 & Secretor & + & - & + & Activo \\
\hline 13 & Proliferativo & - & - & - & Inactivo \\
\hline 14 & Secretor & - & - & - & Inactivo \\
\hline 15 & Proliferativo & - & - & ++ & Activo \\
\hline 16 & Secretor & - & + & ++ & Activo \\
\hline 17 & Secretor & - & - & - & Inactivo \\
\hline 18 & - & +++ & - & - & Activo \\
\hline 19 & Proliferativo & - & - & ++ & Activo \\
\hline 20 & Secretor & $++t$ & ++ & - & Activo \\
\hline 21 & Secretor & - & +++ & + & Activo \\
\hline 22 & Secretor & $+t+$ & $++t$ & + & Activo \\
\hline 23 & Secretor & +++ & $++t$ & + & Activo \\
\hline 24 & Proliferativo & - & - & ++ & Activo \\
\hline 25 & Proliferativo & - & - & + & Activo \\
\hline
\end{tabular}

Signos de clasificación:

+ Escasa; ++ Moderada; +++ Intensa; - Negativa. 
La reacción decidual que se encontró en el $44 \%$ de los casos es ligeramente superior a la encontrada por otro autores; las alteraciones estructurales en un $60 \%$ y las alteraciones morfológicas en un $72 \%$ de todos los casos son superiores a los de otros autores.

Si observamos que los casos 13,14 y 17 corresponden a embarazos ectópicos cuyas vellosidades estaban hialinizadas y en los que posiblemente se habia restablecido el ciclo normal, los 22 casos restantes presentan alteraciones que permiten insinuar la probabilidad de embarazo ectópico.

Si descartamos los casos mencionados quedarían 22 en los cuales considerando reacción decidual y alteraciones morfólogicas y estructurales la posibilidad de embarazo ectópico subiría al $100 \%$.

Otra observación que creemos interesante es que no hay paralelismo entre la reacción decidual y las alteraciones estructurales y especialmente morfológica ccmo en los casos $5,7,8,11,15,16,18$, $19,20,21,24$ y 25 . Total 12 casos que rcpresentan un $48 \%$.

Nuestros resultados como lo demuestra el cuadro No 2 difieren de los de Arias Gutiérrez y Thorkild por una mayor incidencia de alteraciones en el tanto por ciento global donde hubo marcada significación estadistica:

\section{Cuadro No 2}

Comparación Porcentual por Autores, de Resultados Obtenidos en Trabajos Sobre "Alteraciones Endometriales de Embarazo Ectópico"

\begin{tabular}{||c|c|c|c|}
\hline \multicolumn{1}{|c|}{ Autores } & $\begin{array}{c}\text { No de casos } \\
\text { estudiados }\end{array}$ & $\begin{array}{c}\text { Reacción decidual } \\
\%\end{array}$ & $\begin{array}{c}\text { Reacciónó decidual y } \\
\text { alteraciones morfolo- } \\
\text { gicas y estructurales } \\
\%\end{array}$ \\
\hdashline Arias & 32 & $36.3 \%$ & $63.6 \%$ \\
\hdashline Thorkild & Ignorados & $38.0 \%$ & $56.0 \%$ \\
\hline Chaild-Takano & 25 & $44.0 \%$ & $88.0 \%$ \\
\hline \hline
\end{tabular}

Consideramos que esta diferencia se debe a que Arias toma endometrios obtenidos desde 70 hasta 0 días de la intervención y que el porcentaje de tejido corial inactivo es alto en 22 casos de su serie en los que el $57.2 \%$ de las vellosidades estaban totalmente fibrozadas o necrosadas y el $42.8 \%$ estaban parcialmente fibrozadas o necrosadas. 
En nuestro material, insistimos nuevamente, descartamos todos los endometrios obtenidos 26 días antes de la intervención y los casos en que no se comprobó plenamente el estado de vitalidad del tejido corial.

\section{COMENTARIOS}

Consideramos que el trabajo fundamental de Arias en el año de 1954 despertó la curiosidad de varios autores en la investigación de estas alteraciones en el embarazo ectópico. Así Brux J. y Vaissade, Pildes y Wheler, Thorkild Frederiksen y Arias Stella Javier, las describen en 1957.

Corresponde pues a Arias Stella el privilegio de su descripción y hallazgo en el embarazo interrumpido, la mola, el coriocarcinoma.

Respecto al hallazgo de este tipo de alteraciones en embarazo normal, solamente hemos encontrado descrito un caso en una biopsia por aspiración. (1).

Por nuestra parte, hemos tenido la oportunidad de estudiar 5 casos de embarazos normales en histerectomías practicadas como ha bíamos dicho anteriormente por verdaderos y falsos diagnósticos de miomatosis uterina. En los cinco casos estudiados por nosotros encontramos la misma alteración que inicialmente describió Arias en 1954.

Creemos que la etiopatogenia merece un estudio más a fondo y está sujeta a investigaciones posteriores. Por el momento consideramos se ha conseguido un buen auxiliar para el diagnóstico del embarazo ectópico, que merece toda nuestra atención, ya que es alta la incidencia de estas alteraciones que revalúan el concepto del estudio del endometrio como auxiliar en el diagnóstico del embarazo ectópico.

\section{OBSERVACIONES}

1?-En 5 casos de embarazos normales se encontraron las mismas alteraciones endometriales descritas en el embarazo interrumpido, la mola, el coriocarcinoma y el embarazo ectópico.

$2^{\circ}$ - Las alteraciones endometriales descritas, en ausencia de vellosidades coriales, son signos de probabilidad de embarazo ectópico.

$3^{\circ}$ - Las alteraciones mencionadas no parecen guardar paralelismo con la reacción decidual.

\section{BIBLIOGRAFIA}

1- PILDES, R. B., and WHEeleR, J. D., Am. J. Obst. \& Gynec. 73:79, 1957.

2- ARIAS-STElla J. A. M. A. Arch Path. 58: 112.

3- ARIAS-STElla J. A. S. Path. 33: 584.

4 - ARIAS-STELlA J., GARCIA CACERES U. y GUTIERREZ J. J.

5- ARIAS-STElla J., GUTIERREZ J. Rev. Lat. de An. Pat. 1: 81-91, 1957.

6- BRUX J. de, et Vaissade, G. La Press Medical 65: 972, 1957.

7- FREDERIKSEN THORKILD, Acta Obst. et Gynec. Scandinav. 37:86; 1958. 